\title{
Relación de $\mathrm{PM}_{2,5}$ y Enfermedad Respiratoria Aguda en un territorio de Colombia: Modelos Aditivos Generalizados
}

\author{
Hugo Grisales-Romero ${ }^{*}$ orcid.org/0000-0001-5493-0009 \\ Nora Montealegre ${ }^{2}$ orcid.org/0000-0001-5824-4030 \\ Juan Gabriel Piñeros 3 orcid.org/0000-0002-9944-5397 \\ Dorian Ospina 4 orcid.org/0000-0003-2708-5190 \\ Emmanuel Nieto 5 orcid.org/0000-0001-9555-0651
}

Relationship between $\mathrm{PM}_{2.5}$ and Acute Respiratory Disease in a Colombian region: Generalized Additive Models

1. Grupo de Investigación Demografía y Salud, Universidad de Antioquia. Medellín, Colombia.

2. Grupo de Investigación Sistemas de Información, Universidad de Antioquia. Medellín, Colombia.

3. Grupo de Investigación Salud y Ambiente, Universidad de Antioquia. Medellín, Colombia.

4. Universidad de Manitoba. Winnipeg, Canadá.

5. Grupo de Investigación Salud y Sociedad, Universidad de Antioquia, Medellín, Colombia.

\section{Resumen}

Introducción: El efecto deletéreo de material particulado fino exterior sobre la salud respiratoria de la población de niños y de adultos mayores, es de interés en salud pública. Objetivo: Establecer el efecto de la contaminación por Material Particulado de menos de 2,5 $\mu$ m de diámetro $\left(\mathrm{PM}_{2,5}\right)$, sobre la Enfermedad Respiratoria Aguda (ERA) en los menores de 5 y personas de mínimo 65 años, ajustado por variables meteorológicas y climáticas, en los municipios del Área Metropolitana del Valle de Aburrá (Colombia), 2008 a 2015. Materiales y métodos: Estudio ecológico con información de la red de vigilancia de calidad del aire y de registros de prestación de servicios de salud. Se construyeron Modelos Aditivos Generalizados con función de enlace Poisson y suavización spline. Para cada rezago distribuido se calculó la medida de la asociación e intervalo de confianza. Resultados: Los casos de ERA aumentaron significativamente en los menores de 5 años en Envigado y Caldas (43,3\% vs 29,6\%) y en los de 65 y más años, en Medellín (13,2\%) por cada incremento de $10 \mu \mathrm{g} / \mathrm{m}^{3}$ en $\mathrm{PM}_{2,5}$ al día quince a partir de la exposición. Conclusiones: Los eventos diarios respiratorios tuvieron especial frecuencia en Medellín y en municipios de la zona sur.

Palabras clave: Contaminación atmosférica; medio ambiente; enfermedades respiratorias; material particulado; salud. (Fuente: DeCS, Bireme).

\begin{abstract}
Introduction: The harmful effect of fine particulate matter on the respiratory health of child and elderly populations is a concern for public health. Objective: To establish the effect of pollution by less than $2.5 \mu \mathrm{m}$ in diameter $\left(\mathrm{PM}_{2.5}\right)$ particulate matter on Acute Respiratory Disease (ARD) during 2008-2015 in children younger than 5 and adults older than 65 from the Metropolitan Area of the Aburrá Valley (Colombia), adjusting for meteorological and climate variables. Materials and methods: Ecological study with information from the air quality surveillance network and individual records of health providers. Generalized Additive Models were developed using smoothing spline Poisson models. The assessment of the association and confidence intervals were calculated for each distributed lag. Results: For each $10 \mu \mathrm{g} / \mathrm{m}^{3}$ increment in $\mathrm{PM}_{2,5}$ and the day 15 post-exposure, ARD cases increased significantly in populations who are younger than 5 and older than 65 in Envigado and Caldas (43.3\% vs. 29.6\%) and Medellín (13.2\%), respectively. Conclusions: Daily respiratory events had a special frequency in Medellín and the municipalities of the southern region.
\end{abstract}

Keywords: Air pollution; environment; respiratory diseases; particulate matter; health. (Source: DeCS, Bireme).

\footnotetext{
*Autor de correspondencia

Hugo Grisales-Romero

e-mail: hugo.grisales@udea.edu.co
} 


\section{Introducción}

Según la Organización Mundial de la Salud (OMS), cerca 7 millones de muertes prematuras en el mundo fueron atribuidas a la contaminación del aire, de las cuales alrededor del 88\% ocurrieron en países de ingresos bajos y medios de acuerdo con estimaciones del año 2016. En las Américas, la contaminación del aire ambiental es un importante riesgo y según estimaciones se le atribuyeron aproximadamente 249 mil muertes prematuras $(1)$.

De acuerdo con el Índice de Desempeño Ambiental de 2018, la mala calidad del aire es la mayor amenaza ambiental para la salud pública; enfermedades relacionadas con la contaminación del aire contribuyeron al 65\% de los años de vida ajustados por discapacidad (AVAD) relacionados con el medio ambiente en el 2016(2). En el año 2019, en un análisis de la carga de la enfermedad atribuible a la contaminación del aire, se reportó que el material particulado fino era el cuarto factor de riesgo de la mortalidad y discapacidad global y causa de cerca de 6,7 millones de muertes anuales(3).

Existe amplia evidencia sobre los impactos de la contaminación ambiental del aire en los sistemas respiratorio y circulatorio, generado principalmente por material particulado de diámetro menor a 2,5 micrones $\left(\mathrm{PM}_{2,5}\right)$ y menor a 1,0 micrones $\left(\mathrm{PM}_{1,0}\right)$, gases como el ozono $\left(\mathrm{O}_{3}\right)$, el monóxido y el dióxido de nitrógeno $\left(\mathrm{NO}\right.$ y $\left.\mathrm{NO}_{2}\right)$. Entre las causas de mortalidad global asociada a la contaminación del aire se reportan la cardiopatía isquémica $(35,9 \%)$, el accidente cerebrovascular $(21,1 \%)$, la enfermedad pulmonar obstructiva crónica (20,4\%), las infecciones respiratorias agudas $(15,9 \%)$ y cáncer pulmonar y de vías respiratorias $(6,9 \%)^{(4)}$.

Se reconocen algunos mecanismos fisiopatológicos asociados a la exposición de corto plazo y de carácter inusitado, y a la exposición acumulativa de largo plazo, los cuales contribuyen en la generación de las enfermedades o el agravamiento de los síntomas. El $\mathrm{PM}_{2,5}$ es considerado el más peligroso de los contaminantes del aire debido a su mayor capacidad de penetración en el tracto respiratorio inferior, y según algunas fuentes, incluso más profundas, es decir, en los alvéolos(5). El PM ${ }_{2,5}$ está compuesto por iones inorgánicos, nítricos y residuos de ácidos clorhídricos, así como metales alcalinos, incluidos los cationes de potasio y sodio, iones de amonio, carbono orgánico y carbono elemental. Estos componentes actúan mediante diferentes mecanismos que incluyen: 1) daño tisular directo; 2) activación de respuestas inflamatorias agudas y crónicas responsables de la generación de radicales libres de oxígeno y el daño de epitelio pulmonar y la remodelación de la microarquitectra del pulmón; 3) deterioro de la respuesta de inmunidad celular, caracterizada por la disminución de la secreción de citocinas responsables de la señalización celular para la respuesta ante agentes infecciosos; y 4) proceso de genotoxicidad y mutagenicidad en el parénquima pulmonar(6,7).

En Colombia, ha sido usual el reporte de relación entre contaminación atmosférica por $\mathrm{PM}_{1,0}$, y eventos respiratorios, circunscrito a territorios específicos. Para el año 2016, se estimó que las muertes y carga atribuible a la contaminación de aire fueron mayores con respecto a otros factores de riesgo ambientales, destacándose la mortalidad por enfermedades isquémicas del corazón $(290,15$ por cada cien habitantes) y enfermedad pulmonar obstructiva crónica (143,99 por cada cien mil habitantes)(8). El Banco Mundial (BM), ha referido que alrededor de 5000 muertes y 65 millones de AVADs anuales, con un costo cercano a 5,7 trillones de pesos, fueron ocasionados por la contaminación del aire extradomiciliario en Colombia, donde Bogotá y el Área Metropolitana del Valle de Aburrá (AMVA) aportaron más del $75 \%$ de la mortalidad atribuible; por ello, se recomendó la realización de estudios epidemiológicos de mayor alcance para establecer la relación entre calidad del aire y la salud(9).

En comparación con otros factores de riesgo, en el país existen pocos estudios que incluyan las relaciones entre los contaminantes del aire ambiental y los eventos respiratorios. Entre los estudios se destacan principalmente aquellos que abordan el incremento o aparición de sintomatología respiratoria como tos, sibilancias y congestión nasal, entre otros ${ }^{(10,11)}$. La mayoría de los estudios han sido realizados en población menor de 15 años, incluso aquellos en los que se tratan eventos específicos identificados a partir de fuentes secundarias, o por encuestas autodiligenciadas ${ }^{(12)}$. Se destaca el estudio ecológico multiciudad reciente de RodríguezVillamizar et al.(13), quienes reportaron aumento significativo en las visitas a los servicios de urgencias por enfermedades respiratorias en niños entre 5 y 9 años, asociado a los incrementos de $10 \mu \mathrm{g} / \mathrm{m}^{3}$ de $\mathrm{PM}_{1,0}, \mathrm{PM}_{2,5}$ y NO $\mathrm{N}_{2}$. 
En el ámbito local, el ente ambiental del Área Metropolitana del Valle de Aburrá (AMVA), ha reconocido el deterioro en la calidad del aire en las dos últimas dos décadas por el incremento de las concentraciones de material particulado y el riesgo inherente de mayor exposición a enfermedades que son un peligro para la salud de la población, especialmente ante los incrementos en las concentraciones de $\mathrm{PM}_{2,5}$, en principalmente por su capacidad de ocasionar eventos agudos de carácter infeccioso de manera prevalente en los grupos sensibles, lo que exige su conocimiento.

La Política Integral de Salud Ambiental en Colombia, reconoce los impactos poblacionales, especialmente en grupos con mayor exposición y/o susceptibilidad y ha incluido, además, como propósito, el alcance de un medio ambiente sano como determinante para el logro del derecho a la salud(14); en esta línea, la consolidación de un programa de vigilancia epidemiológica en calidad del aire, bastión del Plan Integral de Gestión de Calidad del Aire - PIGECA(15) en su eje temático de "Generación, Aprovechamiento y Fortalecimiento del Conocimiento Científico y la Tecnología", permitirá realizar acciones por parte de quienes toman las decisiones en salud pública en la formulación de estrategias orientadas a su mitigación. El objetivo del presente estudio fue determinar la relación entre las concentraciones de $\mathrm{PM}_{2,5}$ en $\mu_{\mathrm{g}} / \mathrm{m}^{3}$ y la morbilidad por Enfermedad Respiratoria Aguda (ERA) en los menores de cinco y mayores de 65 años en los municipios de la región del Valle de Aburrá entre los años 2008-2015, con el fin de aportar información científica que permita la adopción de estrategias de intervención encaminadas a la prevención de la salud y al fortalecimiento de los sistemas de alerta temprana.

\section{Materiales y métodos}

\section{Diseño, población y lugar de estudio}

Se realizó un estudio ecológico de series de tiempo con el día como unidad temporal y el municipio como unidad de observación. La población de estudio fueron los menores de 5 años y las personas con 65 años o más, residentes en los 10 municipios de la región del Valle de Aburrá (Barbosa, Bello, Caldas, Copacabana, Envigado, Girardota, Itagüí, La Estrella, Medellín y Sabaneta). La elección de los dos grupos de edad se sustentó en la revisión de literatura, que confirma que son grupos susceptibles de afectación respiratoria por los contaminantes atmosféricos $(16,17)$.

\section{Datos de morbilidad}

La información de los casos observados de eventos respiratorios en los servicios de consulta externa, urgencias y hospitalización, se obtuvieron de los Registros Individuales de Prestación de Servicios (RIPS), suministrados por la Secretaría Seccional de Salud y Protección Social del Departamento de Antioquia (SSSPSA). Los casos se identificaron según su lugar de residencia mediante un protocolo de anonimización para asegurar la confidencialidad de los usuarios de los servicios.

Se identificaron los conteos diarios de casos nuevos de visitas a los servicios de urgencias, consulta externa y hospitalización con diagnósticos por ERA de tipo infeccioso según la Clasificación Estadística Internacional de Enfermedades y Problemas Relacionados con la Salud - CIE 10 versión 2015 (Códigos J00-J06/J09-J18/J20-J22) por municipio, grupo de edad y sexo. Según la información suministrada, los RIPS tienen una cobertura del 90\% o más, que sumado al hecho de obtener información de los distintos servicios de atención, permitieron asumir que se contaba con toda la información disponible sobre los casos diarios de ERA para los municipios de la región.

\section{Preparación de los datos del contaminante y de las variables climatológicas}

Los datos ambientales, meteorológicos y climatológicos medidos a nivel de unidades geopolíticas, fueron facilitados por el Sistema Inteligente de Información y la Red de Vigilancia de la Calidad Aire (REDAIRE) del AMVA y por el Instituto de Hidrología, Meteorología y Estudios AmbientalesIDEAM. Se obtuvo información del material particulado de $2,5 \mu \mathrm{m}$ que provenía de 19 estaciones de monitoreo automáticas y semiautomáticas, entre el $1^{\text {o }}$ de abril de 2008 y el 31 de diciembre de 2015 . La información ausente de la concentración diaria de $\mathrm{PM}_{2,5}$ fue estimada previamente, mediante la imputación múltiple por redes neuronales, para luego proceder a ensamblar las diversas series del contaminante mediante técnicas diseñadas para tal fin. En total para el proceso de correlación, se obtuvo información de las 33 estaciones fijas que medían la concentración de $\mathrm{PM}_{2,5}$; para tal efecto, se consideró que en los municipios que conforman el AMVA, los picos de contaminación están asociados a la época lluviosa, con alta pluviosidad y nubosidad; dada esta situación, para el ensamblaje se utilizaron tres estrategias: la primera fue la correlación por ventanas 
móviles(18) que permitió la valoración de la relación existente entre las series de $\mathrm{PM}_{2,5}$ en ventanas de tiempo mensual, con el fin de determinar una serie única de $\mathrm{PM}_{2,5}$ por unidad territorial; para este fin, en cada municipio se evaluaron las correlaciones de Spearman entre $\mathrm{PM}_{2,5}$, en períodos móviles de un mes mediante un algoritmo diseñado para tal fin.; la segunda fue la entropía de la información para la correlación de series de tiempo, basada en la distancia Bhattacharya-Hellinger-Matusita(19) que mide la incertidumbre de una fuente de información, que puede usarse como una técnica para medir correlación cruzada no lineal para series de tiempo continuas y que permite determinar la cercanía relativa entre dos conjuntos de datos; la tercera, fue la combinación o ensamblaje de las series que permitió aprovechar la información disponible de cada serie de $\mathrm{PM}_{2,5}$ con el fin de construir una serie de tiempo que represente de manera única el comportamiento del contaminante por municipio, recurriéndose a los modelos no lineales de efectos mixtos o modelos jerárquicos no lineales(20); los cuales realizan la combinación no lineal de los conjuntos de datos disponibles, para así obtener una serie de tiempo representativa de $\mathrm{PM}_{2,5}$ para todas las estaciones.

Con respecto a las variables meteorológicas, se consideraron la precipitación $(\mathrm{mm})$, la humedad relativa (\%) y la temperatura $\left({ }^{\circ} \mathrm{C}\right)$ para todo el periodo de interés, dada la importancia del clima como factor del ecosistema y por su reconocida influencia en el aumento de las concentraciones de los gases de Efecto Invernadero (GEI)(21) en la atmosfera terrestre, que aunado al efecto de los contaminantes criterio, como el $\mathrm{PM}_{2,5}$, podría ser desencadenante de eventos dañinos en la salud de la población, especialmente en los grupos más sensibles. No se obtuvo información de otras variables climáticas que tienen protagonismo en el ecosistema como la presión atmosférica, la velocidad del viento y la nubosidad, por cuanto el porcentaje de información ausente excedió el 50\% para cada una de ellas, y para el cálculo de los parámetros estadísticos es necesario contar con al menos el 75\% de información válida.

\section{Análisis estadístico}

Se calcularon los indicadores de resumen clásicos para los datos diarios de $\mathrm{PM}_{2,5}$ y sus excedencias; para el cálculo de estas, se categorizaron las series de tal forma que 0 se asignó a todos los valores menores al valor del umbral diario de la norma(22), y 1 para los valores por encima de éste, que corresponde a un día de excedencia; luego, para cada año, se sumaron valores de la serie $(0$ y 1$)$. Las excedencias para cada año se calcularon de acuerdo con la siguiente expresión:
(1)
$=\frac{\begin{array}{c}\text { Porcentaje de excedencias diarias } \\ \text { Días de excedencias } * 100 \%\end{array}}{\text { Días de excedencias }+ \text { Días de no excedencias }}$

Para estimar la relación conjunta del número de casos por ERA en un periodo de tiempo dado, t,-variable dependiente de recuento, $Y_{t}$ - en función de variables meteorológicas y ambientales- $\mathrm{X}-$, se utilizaron modelos semiparamétricos que permiten la inserción explícita de una componente paramétrica, que incluye a la exposición de interés-el contaminante-, y términos no paramétricos para otros predictores. Entre los modelos semiparamétricos, el más utilizado es el Modelo Aditivo Generalizado (GAM, por sus iniciales en inglés) donde se supone que el log t es aditivo en sus predictores, pero no necesariamente lineal(23). El uso de GAM en la contaminación del aire y la salud se ha convertido en un método estándar en esta área(24,25).

Dado que se modela $Y_{t}$ en presencia de posibles factores de confusión, el modelo más comúnmente utilizado es el loglineal de Poisson, en el cual $Y_{t}$ se distribuye Poisson con media $\mu_{t}$ y $\log \mu_{t}$ como predictor lineal. Este generalmente incluye términos para la exposición de interés (esto es, $\mathrm{PM}_{2,5}$ ) y varios factores potenciales de confusión. El modelo es de la forma:

$$
\begin{gathered}
Y_{t} \sim \operatorname{Poison}\left(\mu_{t}\right), \text { con } \\
\log \mu_{t}=\alpha+\beta_{x_{t-l}}+\eta_{\text {confusores medidos }_{t}} \\
+\eta_{\text {confusores no medidos }}
\end{gathered}
$$

donde el contaminante $x_{t-l}$ podría incluirse en el modelo en el rezago 1. El objetivo es la cuantificación parámetro $\beta$ que es el log del riesgo relativo, y $100 \times\left(e^{\beta}-1\right)$ mide el porcentaje de incremento en las defunciones/casos por cada incremento de una unidad en el contaminante(26). 
Se incluyeron una a una las covariables y factores con diferentes grados de libertad para evaluar el aporte de cada una de ellas en la explicación del modelo; la calidad de la estimación del suavizamiento y las estructuras de las tendencias de las covariables se valoraron con alisamiento Thin Plate Regression Spline, cuya aproximación numérica son los grados de libertad estimados, que relacionan el grado de alisamiento y son indicadores o scores de nolinealidad del efecto(27).

Los modelos explicativos definitivos se eligieron utilizando el criterio de información de Akaike, advirtiéndose que el contaminante, como exposición de interés, se incluyó al final de la modelación. De acuerdo con el criterio de expertos, se rezagó la exposición de interés, es decir, el contaminante, de modo que el efecto de éste sobre la oportunidad de enfermar, pudiera ocurrir en el día concurrente, de 0 a 3, de 0 a 7 y de 0 a 15 días (método de rezagos distribuidos). El incremento de la oportunidad de enfermar se interpretó como un porcentaje, exponenciando los coeficientes de las variables que hacían parte de la estructura paramétrica del modelo, acompañado de su respectivo intervalo de confianza del 95\%. La capacidad explicativa del modelo se valoró con el coeficiente de determinación ajustado de $M c F a d e l l-R^{2}$. La consulta de los datos se realizó con el software PostgreSQL 9.6.2 y para la obtención de los resultados se utilizó el paquete estadísticos $R$ versión 3.4.4.

\section{Consideraciones éticas}

Esta investigación se consideró sin riesgo según la Resolución 8430 de 1993 del Ministerio de Salud de Colombia y fue avalada por el Comité de Ética de la
Facultad Nacional de Salud Pública de la Universidad de Antioquia.

\section{Resultados}

Se extrajeron 720 archivos que contenían 113.424.300 registros; en los procesos informáticos de transformación y carga de la información se identificaron 3.487.304, eventos relacionados con la contaminación del aire por material particulado, de los cuales, para ERA, en menores de 5 años y mayores de 65 años, fueron del 23\% (770.941 casos) en el periodo 2008-2015, registrados en los municipios del Área Metropolitana del Valle de Aburrá. Los casos se identificaron según un número asignado por el protocolo de anonimización para asegurar la confidencialidad de los usuarios de los servicios de consulta externa (AC), urgencias (AU), hospitalización (AH) y el archivo de usuario de servicios (US). De los 770.941 casos de ERA seleccionados, el $75,1 \%$ fueron del municipio de Medellín. En todos los municipios se observó que, con relación a los días de la semana, prevaleció el día domingo con la menor cantidad promedio diaria de casos y cuando el día era feriado o festivo, la cantidad de casos de enfermedad respiratoria fue mayor.

De acuerdo con las guías de la calidad del aire de la Organización Mundial de la Salud (OMS), en el periodo de estudio se encontró que el promedio diario de $\mathrm{PM}_{2,5}$ excedió el límite máximo permisible, de $25 \mu \mathrm{g} / \mathrm{m}^{3}$ en todos los municipios del AMVA con excepción de Bello y Girardota; hubo un patrón de presentación de mayores excedencias en los años 2009 y 2015 (Tabla 1).

Tabla 1. Indicadores de resumen de las mediciones diarias de $\mathrm{PM}_{2,5}$ en $\mu \mathrm{g} / \mathrm{m}^{3}$.Municipios del Área Metropolitana del Valle de Aburra. 2008-2015

\begin{tabular}{lrrrrr}
\hline \multicolumn{1}{c}{ Municipio } & $\bar{X}$ & S & Q2 & RI (Q1: Q3) & Mayor\% de Excedencias (Año) \\
\hline Bello & 23,8 & 5,5 & 22,9 & $20,3: 27,0$ & \\
Caldas & 27,8 & 10,3 & 26,8 & $21: 0: 32,5$ & $70,4(2015)$ \\
Copacabana & 37,8 & 11,1 & 37,0 & $30,3: 44,0$ & $28,2(2009)$ \\
Envigado & 25,1 & 6,6 & 24,6 & $20,6: 28,8$ & $47,4(2013)$ \\
Girardota & 23,6 & 13,2 & 19,8 & $16,7: 25,9$ & $20,7(2015)$ \\
Itagüí & 29,7 & 8,9 & 28,5 & $24,1: 33,4$ & $80,5(2009)$ \\
La Estrella & 47,9 & 13,1 & 47,1 & $40,0: 53,2$ & $99,5(2015)$ \\
Medellín & 35,6 & 9,7 & 34,1 & $29,7: 39,4$ & $99,2(2015)$ \\
\hline $\bar{X}:$ Prom
\end{tabular}

$\bar{X}$ : Promedio diario; S: Desviación estándar; Q2: Mediana; RQ: Rango intercuartil; Q1: Primer cuartil; Q3: Tercer cuartil 
Se construyeron los GAM para el número de casos de ERA ajustados por los factores significativos y por las covariables suavizadas (como se indica en el pie de la Tabla 2). Posteriormente se realizó el ajuste $\mathrm{PM}_{2,5}$, como variable de exposición. En los menores de 5 años, los mayores incrementos de los casos diarios de ERA, significativos, se estimaron en los municipios de Envigado (43,3\%), Girardota $(37,2 \%)$, Caldas (29,6\%), La Estrella (25,2\%) y Medellín (18,3\%) al día quince de exposición, por cada aumento de 10 $\mu \mathrm{g} / \mathrm{m}^{3}$ en $\mathrm{PM}_{2,5}$, ajustado por los factores y covariables que se indican en el pie de página de la Tabla 2. Con respecto a las personas de 65 y más años, la oportunidad de incremento del porcentaje de casos de ERA por cada aumento de $10 \mu \mathrm{g} / \mathrm{m}^{3}$ en $\mathrm{PM}_{2,5}$, fue significativa en los municipios de Medellín, Caldas y La Estrella, con estimaciones, hasta el día quince de exposición de 13,2\%, 9,9\% y 5,4\% respectivamente, siempre y cuando los factores y covariables que se indican en el pie de página de la Tabla 2 permanecieran constantes.

Tabla 2. GAM para los eventos respiratorios agudos de tipo infeccioso por grupos de edad y municipio del AMVA con exposición de interés $\mathrm{PM}_{2,5}\left(\mu \mathrm{g} / \mathrm{m}^{3}\right)$

\begin{tabular}{|c|c|c|c|c|c|}
\hline \multirow{2}{*}{ Municipio } & \multirow{2}{*}{ Rezago } & \multicolumn{2}{|l|}{$\% \mathrm{e}^{\beta}$ (IC) } & \multicolumn{2}{|l|}{$\% \mathrm{e}^{\beta}$ (IC) } \\
\hline & & $<5$ años & $R^{2}$ & 65 años o más & $\mathbf{R}^{2}$ \\
\hline \multirow{4}{*}{ Medellín } & 0 & $8,5(8,1-9,0) *$ & 45,2 & $7,5(6,7-8,2) *$ & 27,2 \\
\hline & $0: 3$ & $11,4(10,2-12,7)^{*}$ & 46,0 & $9,4(9,0-9,8)^{*}$ & 27,5 \\
\hline & $0: 7$ & $14,9(13,8-16,1)^{*}$ & 47,6 & $11,2(10,5-11,9)^{*}$ & 27,7 \\
\hline & $0: 15$ & $18,3(17,3-19,3)^{*}$ & 49,0 & $13,2(12,3-14,1)^{*}$ & 28,0 \\
\hline \multicolumn{6}{|l|}{ Zona Sur } \\
\hline \multirow{4}{*}{ Envigado } & 0 & $8,5(2,5-14,8)^{*}$ & 31,0 & $6,3(-1,6-14,9)^{*}$ & 18,3 \\
\hline & $0: 3$ & $8,4(5,2-11,8)^{*}$ & 30,8 & $-0,5(-3,0-2,1)^{*}$ & 18,0 \\
\hline & $0: 7$ & $28,9(23,0-35,0) *$ & 33,8 & $-11,6(-15,2--7,9)^{*}$ & 18,2 \\
\hline & $0: 15$ & $43,3(41,6-57,4)^{*}$ & 36,1 & $-13,3(-17,4--9,1)^{*}$ & 17,4 \\
\hline \multirow{4}{*}{ Itagüí } & 0 & $8(5,9-10,1) *$ & 22,5 & $-0,8(-4,2-2,7)^{* *}$ & 15,4 \\
\hline & $0: 3$ & $9,3(6,8-11,9)$ * & 22,7 & $-2,9(-4,6--1,2)^{* *}$ & 15,6 \\
\hline & $0: 7$ & $11,0(8,6-13,5)$ * & 22,6 & $-3,3(-5,1--1,4)^{* *}$ & 15,5 \\
\hline & $0: 15$ & $14,4(11,4-17,5)$ * & 22,8 & $-3,3(-5,9--0,5)^{* *}$ & 15,5 \\
\hline \multirow{4}{*}{ La Estrella } & 0 & $10,2(5,7-14,6)^{* * *}$ & 8,3 & $7,3(1,7-13,2)^{\circ}$ & 4,0 \\
\hline & $0: 3$ & $15,1(14,8-15,3)$ *** & 10,1 & $4,4(1,6-7,2)^{\circ}$ & 4,9 \\
\hline & $0: 7$ & $18,2(16,7-19,7)$ *** & 10,7 & $4,1(1,1-7,2)^{\circ}$ & 4,9 \\
\hline & $0: 15$ & $25,2(18,3-23,1)^{* * *}$ & 10,5 & $5,4(1,9-9,0)^{\circ}$ & 4,8 \\
\hline \multirow{4}{*}{ Caldas } & 0 & $13,9(10,4-17,4)^{\circ \circ}$ & 15,4 & 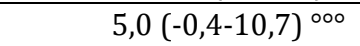 & 3,8 \\
\hline & $0: 3$ & $16,1(13,8-18,4)^{\circ \circ}$ & 15,3 & $6,5(5,9-7,0) \circ \circ$ & 3,6 \\
\hline & $0: 7$ & $22,7(19,7-25,7)^{\circ \circ}$ & 17,8 & $9,4(7,7-11,1) \circ \circ$ & 4,1 \\
\hline & $0: 15$ & $29,6(26,5-33,9)^{\circ \circ}$ & 19,6 & $9,9(7,5-12,4){ }^{\circ \circ \circ}$ & 3,6 \\
\hline \multicolumn{6}{|l|}{ Zona Norte } \\
\hline \multirow{4}{*}{ Girardota } & 0 & $2,7(-17,9-28,5) \dagger$ & 30,1 & $23,4(-7,3-64,3)+\dagger$ & 1,9 \\
\hline & $0: 3$ & $8,0(-0,7-17,4) \dagger$ & 30,4 & $6,2(-7,1-21,4)+\dagger$ & 5,2 \\
\hline & $0: 7$ & $4,1(-5,4-14,4) \dagger$ & 30,3 & $0,1(-13,7-16,1)+\dagger$ & 3,3 \\
\hline & $0: 15$ & $37,2(17,0-60,8) \dagger$ & 41,1 & $-11,4(-25,8-5,8)+\dagger$ & 19,2 \\
\hline
\end{tabular}

*Ajustado por día de la semana, día festivo, día feriado, presencia de brote de influenza, precipitación, humedad relativa y temperatura; ** Ajustado por día de la semana, día festivo, día feriado, presencia de brote de influenza, y humedad relativa; ***Ajustado por día de la semana, día festivo, brote de influenza y humedad relativa; † Ajustado por día de la semana, día festivo y precipitación; †† modelo simple; ${ }^{\circ}$ Ajustada por temperatura; ${ }^{\circ}$ Ajustado por día de la semana, día festivo, precipitación, humedad relativa y temperatura; ${ }^{\circ}$ Ajustado por día de la semana y día festivo

\section{Discusión}

La contaminación del aire es una de las principales preocupaciones en salud pública a nivel mundial, dados sus efectos en la salud humana en el corto y largo plazo. El Valle de Aburrá es una de las regiones más contaminadas de las Américas, donde más del $90 \%$ de los días presenta promedios superiores a 25 $\mu \mathrm{g} / \mathrm{m}^{3(28)}$. Según los estudios de fuentes de emisión, el $80 \%$ de esta contaminación del Valle es debida a la quema de derivados del petróleo, utilizados en el transporte, mientras el $20 \%$ proviene de procesos productivos industriales y fuentes de área(15).

Para este estudio se asumió las guías de calidad del aire de la OMS(22), que establecen los límites máximos 
permisibles, sin embargo, se sabe que Colombia tiene formulados unos estándares estipulados en la Resolución 2254 del 1으 de noviembre de 2017 del Ministerio de Ambiente y Desarrollo Sostenible(29), que discrepan de los límites de la OMS y evidentemente son más laxos para el análisis de los eventos a corto plazo. En los municipios del AMVA, los valores promedios considerados en los ocho años de estudio excedieron los límites permisibles en ocho de los diez municipios considerados, destacándose dicho exceso en todos los de la zona sur.

La topografía compleja de los municipios que conforman el Valle de Aburrá han modificado el patrón de las nubes y sus efectos meteorológicos. En horas de la noche, la contracción del forzamiento de la altura de la capa límite atmosférica sobre la altura de las nubes y la separación de la capa de fricción, ocasiona que la velocidad del viento horizontal en las alturas de las montañas y la cantidad de agua promedio ante la presencia de nubes tengan variadas velocidades. Durante el día, el contraste entre las condiciones meteorológicas y de estabilidad atmosférica en presencia y ausencia de nubes bajas, se agudiza por la disminución en el forzamiento del ciclo diurno sobre las variables atmosféricas, atribuido a las nubes, lo que favorece la acumulación de contaminantes en la superficie, en particular en $\mathrm{PM}_{2,5}{ }^{(30)}$.

Las características anteriores indican que el Valle de Aburrá es una cuenca geográfica estrecha, con desarrollo industrial en todos los municipios, que tiene gran movilidad urbana, importante transporte masivo y demanda energética, aspectos que contribuyen a la generación de $\mathrm{PM}_{2,5}$ de origen antrópico. Lo anterior se manifiesta en el aporte de los automóviles con un 43,5\%, seguido de los camiones con el $16,9 \%$ y el combustible de uso industrial, como el carbón mineral, con el 37\%(31), además de otros factores como la velocidad y dirección del viento, que en los municipios del Valle de Aburrá van de norte a sur y que por lo tanto facilitan el transporte de la contaminación. Asimismo, su geografía adversa en medio de altas montañas, propicia la inversión térmica, con nubes de baja altura y ventilación escasa que se aúna a las ondas del oeste, debido a la intrusión atípica del polvo del Sahara(32) por todo el Atlántico, facilitando las excedencias en $\mathrm{PM}_{2,5}$. Estas características particulares hacen de los municipios del Valle de Aburrá, receptores de emisión de $\mathrm{PM}_{2,5}$.
En este estudio se sustentó la relación de los eventos respiratorios por $\mathrm{PM}_{2,5}$, con la mayor frecuencia de casos a los quince días de haber ocurrido la exposición en los menores de 5 años, destacándose los municipios de Medellín y la zona sur con excepción de Sabaneta, siendo más afectados los municipios de Envigado, Caldas e Itagüí. Cuando se consideraron los adultos mayores, la ERA fue importante en el municipio de Medellín, seguida en orden de magnitud por el municipio de Caldas. Es de resaltar que se reconoce el origen antropogénico del $\mathrm{PM}_{2,5}$ y su contribución para la presentación de la ERA, con factores que facilitan su presentación en los menores de 5 años como son la edad, la exposición al aire libre, la inmadurez fisiológica y anatómica, y en las personas adultas los cambios osteomusculares y la disminución tanto de los mecanismos para expulsar las secreciones respiratorias como de la superficie alveolar(33). Para ambos grupos etarios, la presencia de comorbilidades y el cambio de temporada seca a lluviosa, hacen más difícil el panorama.

Los planes de mitigación y control del PIGECA contienen orientaciones sobre las contingencias con el fin de reducir los niveles de exposición de la población, cuando se presentan los episodios críticos de contaminación del aire, especialmente en los meses de febrero-marzo y septiembre -octubre. Estas medidas incluyen, entre otras, pico y placa ambiental y extendido, la revisión tecno mecánica del parque automotor, las alertas en salud, los días sin carro, la promoción de otros medios de transporte, los planes de reordenamiento territorial, que tienen como finalidad prevenir la presentación de eventos que hacen daño a la salud, como las afecciones respiratorias. Pope et al.(34), evidenciaron que una reducción en la exposición a la contaminación ambiental por partículas finas, contribuyeron a mejoras significativas en la esperanza de vida en los Estados Unidos, durante los años 80's y 90's.

Los estudios de la relación ERA y contaminación son relativamente escasos en el país, por ello este trabajo es un aporte relevante y metodológicamente innovador porque aborda la ERA de tipo infeccioso en población vulnerable desde un enfoque multivariable, donde se escogen como eventos trazadores los respiratorios, de acuerdo con la revisión de las evidencias clínicas y epidemiológicas y el modelo descriptivo de la historia natural de la enfermedad(35). Es la primera investigación en el país con un alcance de 8 años, en 10 municipios, con enfoque 
epidemiológico de la salud ambiental cuyos resultados se suman a la amplia evidencia reportada por la literatura internacional $(9,28,36)$.

La problemática esbozada sustenta el efecto deletéreo del material particulado en general y $\mathrm{PM}_{2,5}$ en particular, en la salud por su capacidad de ocasionar eventos agudos de carácter infeccioso, especialmente en los grupos vulnerables como los menores de 5 años, los de 65 y más años, las gestantes y las personas con comorbilidades, especialmente de tipo respiratorio. Las estrategias de mitigación, mejoramiento de la calidad del aire se han acogido a través de diferentes proyectos tales como el de Sistemas de Alertas Tempranas de Medellín y el Valle de Aburrá (SIATA), que opera la red de monitoreo atmosférico, el inventario de emisiones y el pacto por el mejoramiento de la calidad del aire; este sistema ha permitido la implementación de 21 medidas de las cuales se destacan la no emisión de humo en la industria, la legalización de las actividades de extracción y disposición de desechos de la construcción, el transporte masivo público (metrocable, metroplus, tren metropolitano), el pico y placa ambiental, la renovación del parque automotor público y privado, el plan de descontaminación, así como la generación, aprovechamiento y fortalecimiento del conocimiento científico y tecnológico.

Se avizora que el trabajo mancomunado de los entes ambientes del AMVA tendrá un efecto positivo en la disminución de los eventos adversos a la salud de la población; el Plan Integral de Gestión de la Calidad del Aire del Valle de Aburrá (PIGECA)(15), en sus directrices ha impulsado acciones importantes como la obtención del aval de 13 métodos de medición basados en el Código de Regulaciones Federales de los Estados Unidos de América US-EPA (Environmental Protection Agency), que garantizan la obtención de datos en tiempo real del dióxido de azufre, monóxido de carbono, dióxido de nitrógeno, $\mathrm{O}_{3}, \mathrm{PM}_{1,0}$ y $\mathrm{PM}_{2,5}$. Con ello se prevé avances en la consolidación y producción de la información cuantitativa, para los estudios ambientales requeridos por las autoridades afines competentes de carácter oficial.

\section{Conclusiones}

Los mayores incrementos, significativos, de los casos diarios de ERA, por cada aumento de $10 \mu \mathrm{g} / \mathrm{m}^{3}$ en $\mathrm{PM}_{2,5}$, en los menores de 5 años, se presentaron en los municipios de Envigado (43,3\%), Girardota (37,2\%), Caldas (29,6\%), La Estrella (25,2\%) y Medellín $(18,3 \%)$. En las personas de 65 y más años los mayores incrementos fueron en Medellín (13,2\%), Caldas $(9,9 \%)$ y La Estrella, (5,4\%). En general, el riesgo de presentación diaria de eventos respiratorios, se reflejaron especialmente en los municipios de la zona sur y en la ciudad de Medellín.

\section{Limitaciones}

Dadas las características del diseño fue imposible el control del sesgo ecológico ya que se asumió que la exposición se distribuyó homogéneamente en todo el grupo menor de cinco, 65 y más años, debido a que la medición es a nivel poblacional; además, no se consideraron las interacciones con otros contaminantes denominados de exposición simultánea. Sólo se abordaron los registros que refirieron la demanda atendida, con los RIPS, cuyas principales limitantes estriban en la captación del caso, del registro y la validación del diagnóstico.

\section{Recomendaciones}

Se recomienda avanzar en estudios de tipo individual para explorar y definir la exposición y su gradiente, el riesgo acumulado y los efectos de la contaminación por $\mathrm{PM}_{2,5}$ que permita trascender a la causalidad. También es importante realizar estudios que permitan la georreferenciación tanto de la morbilidad como de la mortalidad por eventos relacionados con la contaminación del aire, no sólo por $\mathrm{PM}_{2,5}$ sino que también incluya los demás contaminantes criterio, lo que permitirá el establecimiento de una línea de base que facilite la ubicación de las residencias y comunidades cerradas de niños y adultos y su posterior seguimiento.

Conflicto de intereses: Ninguno declarado por los autores.

\section{Financiamiento}

Este estudio fue financiado por el programa Ciencia, Tecnología e Innovación, Colciencias, mediante convocatoria 744-2016 contrato No. 633-2017 y por el Área Metropolitana del Valle de Aburrá, contrato CCT 1133 de 2018.

\section{Referencias}

1. Calidad del aire [Internet]. OPS-OMS; 2020 [consultado 2021 Feb 11]. Disponible en: https://www.paho.org/es/temas/calidad-aire 
2. Friedrich MJ. Air Pollution Is Greatest Environmental Threat to Health. JAMA [Internet]. 2018 Mar 20 [consultado 2021 Feb 13];319(11):1085. DOI: 10.1001/jama.2018.2366.

3. GBD 2019 Risk Factors Collaborators. Global burden of 87 risk factors in 204 countries and territories, 1990-2019: a systematic analysis for the Global Burden of Disease Study 2019. Lancet. 2020;396(10258):1223-49. DOI: 10.1016/S0140-6736(20)30752-2.

4. Cohen AJ, Brauer M, Burnett R, Anderson HR, Frostad J, Estep $\mathrm{K}$, et al. Estimates and 25-year trends of the global burden of disease attributable to ambient air pollution: an analysis of data from the Global Burden of Diseases Study 2015. Lancet. 2017;389(10082):1907-18. DOI: 10.1016/S01406736(17)30505-6.

5. Pope CA, Dockery DW. Health effects of fine particulate air pollution: Lines that connect. J Air Waste Manag Assoc. 2006;56(6):709-42.

10.1080/10473289.2006.10464485.

6. Grzywa-Celińska A, Krusiński A, Milanowski J. 'Smoging kills' - Effects of air pollution on human respiratory system. Ann Agric Environ Med. 2020;27(1):1-5. DOI: 10.26444/aaem/110477.

7. Brugha R, Grigg J. Urban air pollution and respiratory infections. Paediatr Respir Rev. 2014;15(2):194-9. DOI: 10.1016/j.prrv.2014.03.001.

8. Observatorio Nacional de Salud. Carga de enfermedad ambiental en Colombia [Internet]. 2018 [consultado 2021 Feb 11]. Disponible en: https://www.ins.gov.co/Direcciones/ONS/Resumenes Ejecutivos/Resumen ejecutivo informe10 Carga de enfermedad en Colombia.pdf

9. Golub E, Klytchnikova I, Sanchez-Martinez G, Belausteguigoitia JC. Environmental Health Costs in Colombia The Changes from 2002 to 2010 [Internet]. Washington, DC: World Bank; 2014 [consultado $2021 \mathrm{Feb}$ 11]. 63 p. 63 Disponible https://openknowledge.worldbank.org/handle/10986/210 96

10. Rodríguez Villamizar LA, Herrera López AB, Castro Ortiz H, Niederbacher Velázquez J, Vera Cala LM. Incidencia de síntomas respiratorios y su asociación con contaminación atmosférica en preescolares: un análisis multinivel. Cad Saude Publica. 2010;26(7):1411-8. DOI: 10.1590/S0102311X2010000700020.

11. Hernández-Flórez LJ, Aristizabal-Duque G, Quiroz L, Medina K, Rodríguez-Moreno N, Sarmiento R, et al. Air pollution and respiratory illness in children aged less than 5 years-old in Bogotá, 2007. Rev salud pública. 2013;15(4):503-16. Disponible en: https://revistas.unal.edu.co/index.php/revsaludpublica/art icle/view/38719

12. Herrera-Torres AM, Echeverri-Londoño CA, Maya-Vasco GJ, Ordoñez-Molina JE. Patologías respiratorias en niños preescolares y su relación con la concentración de contaminantes en el aire en la ciudad de Medellín (Colombia). Rev Ing Univ Medellín [Internet]. 2011;10(19):21-32. Disponible en: https://revistas.udem.edu.co/index.php/ingenierias/article /view/435

13. Rodríguez-Villamizar LA, Rojas-Roa NY, Blanco-Becerra LC, Herrera-Galindo VM, Fernández-Niño JA. Short-Term Effects of Air Pollution on Respiratory and Circulatory Morbidity in Colombia 2011-2014: A Multi-City, Time-Series Analysis. Int
J Environ Res Public Health. 2018;15(8):1610. DOI: 10.3390/ijerph15081610.

14. Plan Decenal de Salud Pública, 2012-2021 [Internet]. Ministerio de Salud y Protección Social. Disponible en: https://www.minsalud.gov.co/plandecenal/Paginas/home 2013.aspx

15. Plan Integral de Gestión de la Calidad del Aire para el Área Metropolitana del Valle de Aburrá (PIGECA 2017-2030) [Internet]. Area Metropolitana del Valle de Aburra; 2017. Disponible

https://www.metropol.gov.co/ambiental/calidad-delaire/Paginas/Gestion-integral/PIGECA.aspx

16. Sevillano E. Niños y ancianos, los más vulnerables a la contaminación por PM2,5. EL PAÍS: Madrid. 2021 Jan 11 [consultado 2021 Feb 27]. Disponible en: https://elpais.com/diario/2011/01/11/madrid/12947486 54_850215.html

17. Vargas S, Onatra W, Osorno L, Páez E, Sáenz 0. Contaminación atmosférica y efectos respiratorios en niños, en mujeres embarazadas y en adultos mayores. Rev UDCA Actual Divulg Científica. 2008;11(1):31-45. Disponible en: http://www.scielo.org.co/pdf/rudca/v11n1/v11n1a06.pdf

18. Shakil S, Keilholz SD, Lee C-H. On frequency dependencies of sliding window correlation. In: Proceedings - 2015 IEEE International Conference on Bioinformatics and Biomedicine, BIBM 2015 [Internet]. Institute of Electrical and Electronics Engineers Inc.; 2015 [consultado 2021 Jun 11]. p. 363-8. Disponible en: https://ieeexplore.ieee.org/abstract/document/7359708

19. Li H. Nonsymmetric Dependence Measures: the Discrete Case. 2015 [consultado 2021 Jun 11]. Disponible en: https://arxiv.org/abs/1512.07945

20. Stegmann G, Jacobucci R, Harring JR, Grimm KJ. Nonlinear Mixed-Effects Modeling Programs in R. Struct Equ Model [Internet]. 2018 Jan 2 [consultado 2021 Jun 11];25(1):1605. DOI: 10.1080/10705511.2017.1396187.

21. IPCC-Intergovernmental Panel on Climate Change. Climate change 2014: synthesis report. In: Contribution of Working Groups I, II and III to the Fifth Assessment Report of the Intergovernmental Panel on Climate Change. Geneva, Switzerland: IPCC [Internet]; 2014 [consultado 2021 Jun 12]. Disponible en: https://www.ipcc.ch/site/assets/uploads/2018/05/SYR_A R5_FINAL_full_wcover.pdf.

22. OMS. Guías de calidad del aire de la OMS relativas al material particulado, el ozono, el dióxido de nitrógeno y el dióxido de azufre: actualización mundial 2005 [Internet]. 2006. [consultado 2021 Feb 24]. 28 p. Disponible en: https://apps.who.int/iris/bitstream/handle/10665/69478 /WHO_SDE_PHE_OEH_06.02_spa.pdf

23. Wood S. Generalized Additive Models: An Introduction with R. 2nd ed. Boca Raton, USA: CRC Press; 2017 [consultado 2021 Jun 16]. Disponible en: https://books.google.es/books?hl=es\&lr=\&id=HLPDwAAQBAJ\&oi $=$ fnd $\& p g=P P 1 \& d q=+$ Generalized + Additive + Models:+An+Introduction+with+R\&ots=NqTIeitBn6\&sig=0152z2ACtvaHu9nSv4lA7D2n_8\#v=onepage\&q\&f=false

24. Hastie TJ, Tibshirani R. Generalized Additive Models. Statistical Models in S [Internet]. Routledge; 1992 [consultado 2021 Jun 14]. Disponible en: https://www.taylorfrancis.com/chapters/edit/10.1201/97 80203738535-7/generalized-additive-models-trevor-hastie

25. Schwartz J. Nonparametric smoothing in the analysis of air pollution and respiratory illness. Can J Stat [Internet]. 1994 
Dec [consultado 2021 Feb 11];22(4):471-87. DOI: $10.2307 / 3315405$.

26. Peng RD, Dominici F. Statistical methods for environmental epidemiology with R: a case study in air pollution and health [Internet]. New York, USA: Springer; 2008 [consultado 2021 Feb 11]. Disponible en: https://dialnet.unirioja.es/servlet/libro?codigo=372584

27. Keller W, Borkowski A. Thin plate spline interpolation. Artic J Geod [Internet]. 2019 [consultado 2021 Feb 27]. DOI: 10.1007/s00190-019-01240-2.

28. Área Metropolitana del Valle de Aburrá. Contaminación atmosférica y sus efectos sobre la salud de los habitantes del Valle de Aburrá. 2008-2015 [Internet]. Medellin, Colombia: Universidad de Antioquia; 2019 [consultado 2021 Feb 13]. 116 p. Disponible en: https://www.metropol.gov.co/ambiental/calidad-delaire/Biblioteca-aire/Reanalisis/ContaminacionAtmosferica_y_sus_Efectos_en_la_Sal ud-AMVA_2019.pdf

29. Ministerio de Ambiente y Desarrollo Sostenible. Resolucion 2254 del 1 de noviembre del 2017 Por la cual se adopta la norma de calidad del aire ambiente y se dictan otras disposiciones [Internet]. 2017 [consultado 2021 Feb 24]. Disponible en: https://www.minambiente.gov.co/wpcontent/uploads/2021/10/Resolucion-2254-de-2017.pdf

30. Velásquez García MP. Caracterización meteorológica de la atmosfera en presencia de nubes bajas sobre zona plana del Valle en el Aburra [Tesis de grado]. Medellin, Colombia: Universidad Nacional de Colombia; 2019 [consultado 2021 Jun 15]. Disponible en: https://www.metropol.gov.co/ambiental/calidad-del-
aire/Biblioteca-aire/InvestigacionSIATA/Tesis-

Caracterizacion-Atmosfera.pdf

31. Porta A, Sanchez EY, Colman Lerner E, Massolo L, Gutierrez M, Orte M, et al. Calidad Del Aire: Monitoreo y modelado de contaminantes atmosféricos. Efectos en la salud pública [Internet]. Buenos Aires, Argentina; Universidad de La Plata; 2018 [consultado 2021 Jun 15]. Disponible en: https://ri.conicet.gov.ar/bitstream/handle/11336/136723 /CONICET_Digital_Nro.14856eb7-05bd-4812-a99e50ed2b7552e4_V.pdf?sequence=5\&isAllowed =y

32. Mendez Espinosa JF, Pinto Herrera LC, Belalcázar Cerón LC. Study of a Saharan Dust Intrusion into the Atmosphere of Colombia. Ing Univ Medellín [Internet]. 2018 [consultado 2021 Feb 24];17(32):17-34. D0I: 10.22395/rium.v17n32a1.

33. Bernstein JA, Alexis N, Barnes C, Bernstein IL, Bernstein JA, Nel A, et al. Health effects of air pollution. J Allergy Clin Immunol [Internet]. 2004 Nov 1 [consultado 2021 Jun 15];114(5):1116-23. DOI: 10.1016/j.jaci.2004.08.030.

34. Pope CA, Ezzati M, Dockery DW. Fine-Particulate Air Pollution and Life Expectancy in the United States. $N$ Engl J Med [Internet]. 2009 Jan 22 [consultado 2021 Feb 13];360(4):376-86. Disponible en: http://www.nejm.org/doi/abs/10.1056/NEJMsa0805646

35. Arouca S. La historia natural de las enfermedades. Rev Cuba Salud Pública [Internet]. 2018 [consultado 2021 Feb 24];44(4):220-8. Disponible en: https://www.scielosp.org/article/rcsp/2018.v44n4/220228/es/

36. Ortega-García JA, Sánchez-Solís M, Ferrís-Tortajada J. Air pollution and children's health. An Pediatr [Internet]. 2018 Aug 1 [consultado 2021 Feb 27];89(2):77-9. DOI: 10.1016/j.anpedi.2018.04.017. 